\title{
Criação intensiva de suínos em confinamento ou ao ar livre: estudo meta-analítico do desempenho zootécnico nas fases de crescimento e terminação e avaliação de carcaça e carne no Longissimus dorsi
}

\author{
Intensive pig production in confinement or outdoor systems: A meta-analytical study of the production \\ performance in the growing and finishing phases and carcass and meat evaluation from the \\ Longissimus dorsi
}

\author{
Alice Bogoni Demori ${ }^{*}$ Paulo Alberto Lovatto $^{\dagger}{ }^{\dagger}$ Ines Andretta $^{\mathrm{I}}$ Marcos Kipper $^{\mathrm{I}}$ \\ Cheila Roberta Lehnen ${ }^{I}$ Aline Remus ${ }^{\mathrm{I}}$
}

\section{RESUMO}

Uma meta-análise foi realizada para avaliar o desempenho e a qualidade de carcaça e carne de suínos em função do sistema de alojamento nas fases de crescimento/ terminação. Foram selecionadas publicações indexadas com resultados de experimentos comparando variáveis de desempenho e de pós-abate de suínos alojados em sistemas intensivos convencionais ou ao ar livre. A base de dados contemplou 27 artigos publicados entre 1997 e 2009. Ao total, foram considerados 2.985 animais e 701 médias na análise de dados. A meta-análise foi realizada através de duas análises sequenciais: gráfica e de variância-covariância. O sistema de criação de suínos ao ar livre aumentou em $9 \%(P<0,05)$ o consumo de ração, reduziu em $2 \%(P<0,01)$ o ganho de peso diário e piorou em $3 \%(P<0,05)$ a conversão alimentar dos animais em relação ao sistema confinado. As características de carcaça (peso de carcaça quente, rendimento, espessura de toucinho e percentual de carne magra) e de carne no Longissimus dorsi (perda por gotejamento e cozimento, $\mathrm{pH}$, temperatura inicial, força de cisalhamento, marmoreio dureza, maciez e suculência) não foram influenciadas $(P>0,05)$ pelo sistema de alojamento dos suínos. Concluiu-se que o sistema de criação influencia no desempenho dos suínos, mas não interfere nas características de carcaça e carne estudadas.

Palavras-chave: alojamento alternativo, desempenho em crescimento e terminação, carcaça, metaanálise, sistema de produção.

\section{ABSTRACT}

A meta-analysis was carried out to study performance and characteristics of carcass and meat in pigs in function of housing system during the growing/finishing period. Indexed publications with results of experiments comparing performance and post-slaughter variables of pigs housed in outdoor or conventional intensive systems were selected. In total, 2.985 animals and 701 averages were considered in the data analysis. Meta-analysis followed two sequential analyses: graphic and variance. The outdoor productive system increased in $9 \%(P<0.05)$ feed intake, reduced in $2 \%(P<0.01)$ daily weight gain and worsened in $3 \%(P<0.05)$ feed conversion ratio of pigs in comparison to animals in the confined system. Characteristics of carcass (hot carcass weight, yield, backfat thickness and lean meat percentage) and Longissimus dorsi meat (drip and cooking loss, $\mathrm{pH}$, the initial temperature, shear force, marbling, hardness, juiciness and tenderness) were not affected $(P>0.05)$ by the housing system. It is concluded that the housing system influences the pig performance, but does not interfere in characteristics of carcass and meat.

Key words: alternative housing, carcass, meta-analysis, growing and finishing performance, productive system.

\section{INTRODUÇÃO}

A expansão da produção suinícola se deu pela intensificação do processo de criação dos animais. O avanço das pesquisas com melhoramento genético, nutrição e manejo substituiu, ao longo da história, os sistemas criatórios ao ar livre por sistemas intensivos com um espaço limitado e alta densidade animal. Entretanto, esse modelo de criação trouxe para discussão os temas relacionados com o bem-estar animal.

Enquanto são buscadas novas formas de defender os direitos dos animais, muitos pesquisadores

IGrupo de Modelagem Animal, Departamento de Zootecnia, Universidade Federal de Santa Maria, 97105-900, Santa Maria, RS, Brasil. E-mail: alice_bd@hotmail.com. *Autor para correspondência. ${ }^{\dagger}$ In memorian. 
têm dedicado seus estudos a determinar quanto e de qual forma o tipo de edificação utilizada para a criação dos suínos influencia no seu desempenho produtivo e na qualidade de carcaça e carne (JONSÄLL et al., 2001; NILZÉN et al., 2001). Entretanto, os resultados observados nestes trabalhos ainda apresentam grande variabilidade, especialmente em função das diferentes condições experimentais utilizadas. Diante desse cenário, o estudo da influência dos sistemas produtivos poderia ser realizado adequadamente através da metaanálise, uma técnica que permite integrar diferentes variáveis, estabelecendo respostas ajustadas à diversidade experimental (LOVATTO et al., 2007). O presente trabalho foi desenvolvido com o objetivo de avaliar o desempenho zootécnico pelas variáveis de consumo de ração, conversão alimentar e ganho de peso; as características de carcaça pelas medidas de peso de carcaça quente, rendimento, espessura de toucinho e porcentagem de carne magra; as características do músculo Longissimus dorsi como dureza, maciez, suculência entre outras variáveis qualitativas de suínos criados em sistemas confinados ou ao ar-livre.

\section{MATERIAL E MÉTODOS}

Foram selecionadas publicações indexadas com experimentos que avaliaram o desempenho e as características de carcaça e carne de suínos em diferentes sistemas de criação. A estratégia de pesquisa dos trabalhos foi a consulta de diferentes bancos de dados digitais. Os principais critérios para a seleção das publicações foram: (a) comparação de sistemas confinados ou ao ar livre; (b) suínos de linhagens comerciais; (c) fases crescimento ou terminação; (d) respostas de desempenho (consumo de ração, ganho de peso e conversão alimentar); e (e) avaliação de características de carcaça e do Longissimus dorsi. Para considerar a qualidade metodológica dos estudos, foram selecionadas apenas publicações de periódicos com fator de impacto superior a 1,4. Após a seleção dos trabalhos e análise exploratória, foram tabuladas as informações relativas ao objetivo de estudo e também outras variáveis (como número de total de animais, número de animais por tratamento, sexo) para permitir a análise descritiva dos estudos inseridos na base. Essas informações foram selecionadas nas seções do material e métodos e dos resultados dos artigos.

A base de dados foi composta por 27 artigos publicados entre 1997 e 2009 (mediana: 2003). Um resumo das principais características dos artigos utilizados para a base de dados é apresentada na tabela 1 .
Dos periódicos utilizados, 52\% foram publicados no periódico Meat Science e 15\% são publicações do Journal of Animal Science. A maior parte dos estudos foi realizada em instituições europeias (78\%). Os dados das publicações incluídas na base totalizaram 2.985 suínos, com média de 36 animais por tratamento. Os animais apresentaram um peso médio inicial de $34,4 \mathrm{~kg}$ $(\mathrm{DP}=22,4)$ e de $108,6 \mathrm{~kg}$ ao abate $(\mathrm{DP}=17,1)$. O número de médias disponíveis para análise foi de 701.

A metodologia para a definição das variáveis dependentes e independentes e para a codificação dos dados seguiu as proposições descritas na literatura (LOVATTO et al., 2007). Algumas codificações foram utilizadas com critérios qualitativos de agrupamento, como recurso para associar grupos homogêneos em determinados critérios e incluí-los nos modelos analíticos como fonte de variação. Nesse particular, a principal codificação utilizada foi para tipo de instalação (sistema confinado ou ao ar livre). Outras codificações foram utilizadas como variáveis moderadoras nas análises, com o objetivo de considerar a variabilidade dos estudos compilados (efeito do artigo - número sequencial específico para cada trabalho da base; efeito inter - código específico para cada tratamento da base; e efeito intra - atribuído aos grupos com medidas repetidas). A meta-análise seguiu duas análises sequenciais: gráfica (para avaliar a qualidade da base e observar a coerência biológica dos dados) e de variância-covariância. As codificações moderadoras (1) geral, intra ou inter e (2) para tipo de instalação foram utilizadas nos modelos para as análises de variância. Outros efeitos importantes (características de ambiente, variáveis nutricionais, sexo e genética dos animais) não foram considerados devido à falta da informação ou de padronização nas publicações da base de dados. As variáveis sensoriais dureza, maciez, suculência e marmoreio foram submetidas ao Teste de KruskallWallis. As análises foram realizadas através do programa Minitab $^{\circledR} 15$ (2006). A medida de dispersão utilizada foi o desvio-padrão residual (DPR), uma vez que caracteriza melhor a variabilidade intra-modelo.

\section{RESULTADOS E DISCUSSÃO}

Os suínos criados em sistemas ao ar livre apresentaram consumo de ração diário 9\% superior $(\mathrm{P}=0,027)$ quando comparados aos animais criados no sistema confinado (Figura 1). Além disso, animais criados em sistemas ao ar livre apresentaram ganho de peso diário $2 \%$ inferior $(\mathrm{P}=0,002)$ e conversão alimentar $3 \%$ pior $(\mathrm{P}=0,035)$ em relação ao grupo confinado.

As interferências negativas observadas no desempenho dos suínos não confinados podem ser 
Tabela 1 - Dados descritivos dos artigos utilizados na base de dados para a meta-análise.

\begin{tabular}{llcccc}
\hline Autor & \multicolumn{1}{c}{ País } & N de médias & N de animais & Peso médio inicial, kg & Peso médio final, kg \\
\hline BRIDI et al. (2003) & Brasil & 14 & 96 & - & - \\
CARRAPISO et al. (2003) & Espanha & 12 & 34 & - & - \\
ENFÄLT et al. (1997) & Suécia & 22 & 102 & - & - \\
GADE (2008) & Dinamarca & 6 & 234 & - & 117,2 \\
GENTRY et al. (2002) & EUA & 32 & 48 & 8,9 & 108,9 \\
GENTRY et al. (2002) & EUA & 44 & 80 & 40,7 & 116,5 \\
GENTRY et al. (2004) & EUA & 20 & 48 & 8,8 & 80,1 \\
GUY et al. (2002) & Inglaterra & 35 & 674 & 29,2 & 110,4 \\
HANSEN et al. (2006) & Dinamarca & 68 & 160 & 40,5 & 81,5 \\
HOFFMAN et al. (2003) & África do Sul & 16 & 24 & 27,7 & 108,5 \\
HOGBERG et al. (2004) & Suécia & 18 & 39 & 22,8 & 102,0 \\
JONSÄLL et al. (2001) & Suécia & 2 & 48 & 60,0 & 89,2 \\
KELLY et al. (2007) & Inglaterra & 14 & 48 & 30,8 & 107,0 \\
LAMBOOIJ et al. (2004) & Holanda & 40 & 131 & - & 108,9 \\
LEBRET et al. (2002) & França & 30 & 184 & 25,0 & 113,1 \\
LEBRET et al. (2006) & França & 68 & 120 & 35,0 & 113,1 \\
LEBRET (2008) & França & 22 & & 30,0 & 105,0 \\
MILLET et al. (2004) & Bélgica & 84 & 77 & - & 110,0 \\
MILLET et al. (2005) & Bélgica & 72 & 120 & 21,0 & 102,0 \\
NILZÉN et al. (2001) & Suécia & 10 & 120 & 26,0 & 108,2 \\
OLSSON et al. (2003) & Suécia & 16 & 80 & 28,0 & 113,0 \\
PUGLIESE et al. (2003) & Itália & 26 & 78 & 79,0 & 131,9 \\
PUGLIESE et al. (2004) & Itália & 10 & 46 & - & - \\
PUGLIESE et al. (2004) & Itália & 14 & 78 & - & 170,0 \\
REY et al. (2006) & Espanha & 4 & 32 & 42,2 & \\
STRUDSHOLM et al. (2005) & Dinamarca & 16 & 196 & \\
TROMBETTA et al. (2009) & Itália & 16 & 40 & \\
\hline
\end{tabular}

relacionadas com o maior gasto energético dos animais mantidos nestes sistemas criatórios (MILLET et al., 2005; LEBRET et al., 2006, 2008). O maior estímulo ao comportamento exploratório (STRUDSHOLM \& HERMANSEN 2005; HANSEN et al., 2006) e a influência do meio ambiente (LAMBOOIJ et al.,2004) também são fatores que devem ser considerados para explicar a interferência do sistema criatório sobre o desempenho. O fator temperatura, sobretudo, pode ter grande influência sobre o desempenho dos animais (LEBRET et al., 2002). Os sistemas confinados permitem, em geral, maior controle antrópico sobre a temperatura ambiente. Nesse contexto, é possível esperar que animais criados ao ar livre dispensem esforços na busca da manutenção de seu conforto térmico.

A tabela 2 apresenta as características de carcaça e de carne em função do alojamento. As características de peso de carcaça quente $(\mathrm{P}=0,203)$ e rendimento de carcaça $(\mathrm{P}=0,900)$ não foram influenciados pelo sistema de criação dos animais. Resultados semelhantes foram observados nos estudos de GUY et al. (2002), REY et al. (2006) e KELLY et al. (2007). O percentual de carne magra ( $\mathrm{P}=0,361)$ e as espessuras de toucinho também não foram influenciados pelo sistema de criação $(\mathrm{P}=0,627$ para a medida na primeira costela; $\mathrm{P}=0,849$ na última costela; e $\mathrm{P}=0,692$ na última lombar), sendo que resultados semelhantes foram descritos por MILLET et al. (2004) e HOGBERG et al. (2004). As características de dureza ( $\mathrm{P}=0,819)$, maciez $(\mathrm{P}=0,364)$ e suculência $(\mathrm{P}=0,223)$ também não diferiram entre os sistemas, corroborando dados de GENTRY et al. (2002a) e HOFFMAN et al. (2003).

As perdas de água por gotejamento ( $\mathrm{P}=0,252)$ e por cozimento $(\mathrm{P}=0,855)$ do Longissimus dorsi não diferiram nos animais confinados e nos criados ao ar livre. $\mathrm{O}$ pH inicial $(\mathrm{P}=0,526)$ e final $(\mathrm{P}=0,446)$ e o marmoreio $(\mathrm{P}=0,647)$ também não foram influenciados pelos sistemas de criação estudados. Resultados semelhantes em relação ao $\mathrm{pH}$ foram observados em estudos anteriores (BRIDI et al. 2003; PUGLIESE et al., 2004a). Outros autores, entretanto, observaram diferenças no pH da carne dos suínos em função dos sistemas de produção, a exemplo de GADE (2008) que relatou valores de $\mathrm{pH}$ mais elevado para suínos confinados.

Ciência Rural, v.42, n.7, jul, 2012. 

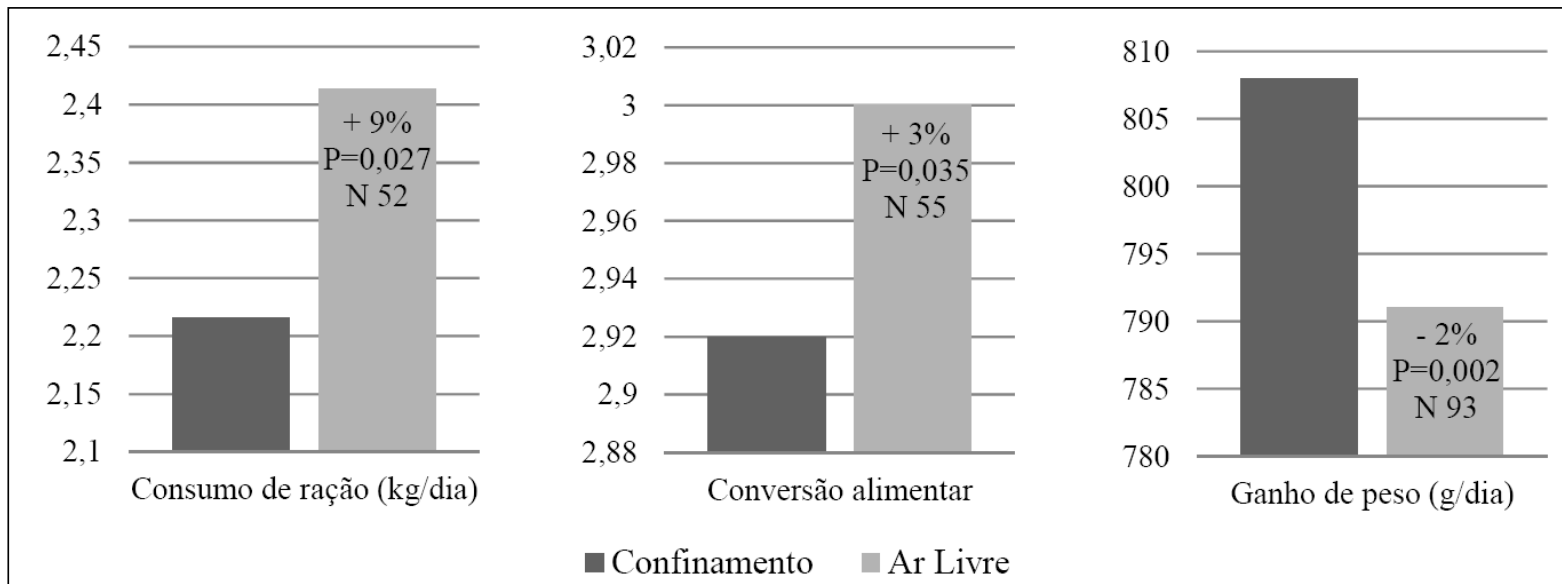

Figura 1 - Desempenho de suínos em função do tipo de alojamento. P: probabilidade; N: número de médias utilizadas para cada análise.

Não foram verificadas diferenças entre os tratamentos para temperatura inicial $(\mathrm{P}=0,311)$ e força de cisalhamento $(\mathrm{P}=0,881)$. Os resultados obtidos nesta meta-análise diferem dos observados em estudos anteriores, nos quais os suínos criados ao ar livre apresentaram menores valores para $\mathrm{pH}$ final, capacidade de retenção de água e marmoreio e maiores valores para força de cisalhamento em comparação com animais mantidos em confinamento durante as fases de crescimento e/ou terminação (ENFÄLT et al., 1997; CARRAPISO et al.,2003; OLSSON et al., 2003; PUGLIESE et al., 2003 e 2004b). Como a influência do sistema produtivo em variáveis de pós-abate não foi observada através desta meta-análise, é provável que as alterações observadas em trabalhos anteriores estivessem relacionadas com características

Tabela 2 - Características de carcaça e carne de suínos em função do tipo de alojamento.

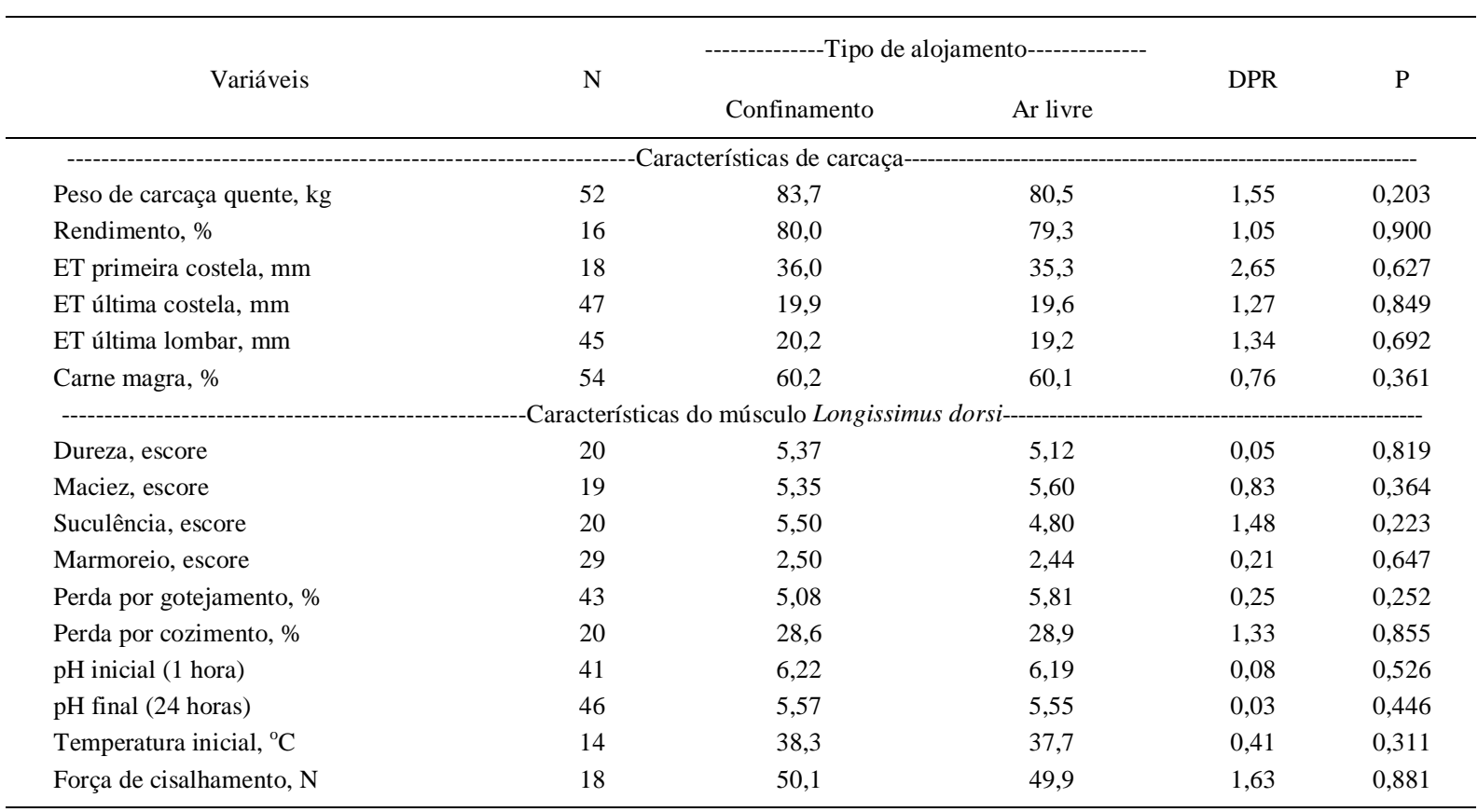

ET: espessura de toucinho. DPR: desvio padrão residual. P: probabilidade indicada por ${ }^{\mathrm{ns}} \mathrm{P}>0,05$.

Ciência Rural, v.42, n.7, jul, 2012. 
específicas dos experimentos, como genética ou condições ambientais. Tais alterações, entretanto, não se mantiveram em uma análise global e ajustada.

A meta-análise realizada neste trabalho permitiu avaliar sistematicamente as alterações no desempenho de suínos em diferentes sistemas de criação. Os resultados obtidos apontaram para a possibilidade de novas abordagens para os experimentos envolvendo a comparação entre sistemas de produção. Em nosso estudo, diversas variáveis não foram passíveis de análise, especialmente por falhas na descrição dos ambientes experimentais utilizados nos trabalhos da base de dados. A possível influência de fatores ambientais e nutricionais, por exemplo, seria um importante avanço para novos estudos comparando os sistemas produtivos.

\section{CONCLUSÃO}

Suínos criados ao ar livre apresentam maior consumo de ração, menor ganho de peso e pior conversão alimentar em relação aos criados em sistemas de confinamento. As principais características de carcaça e do músculo Longissimus dorsi de suínos não diferem entre os sistemas de alojamento confinado e ao ar livre.

\section{REFERÊNCIAS}

BRIDI, A.M. et al. Efeito do genótipo halotano e de diferentes sistemas de produção na qualidade da carne suína. Revista Brasileira de Zootecnia, v.32, n.6, p.1362-1370, 2003. Disponível em: <http://dx.doi.org/S1516-35982003000400021>. Acesso em: 20 jun. 2011. doi: S1516-35982003000400021.

CARRAPISO, A.I. et al. Effect of crossbreeding and rearing system on sensory characteristics of iberian ham. Meat Science, v.65, p.623-629, 2003. Disponível em: <http:// dx.doi.org/10.1016/S0309-1740(02)00256-5>. Acesso em: 26 jun. 2011. doi: 10.1016/S0309-1740(02)00256-5.

ENFÄLT, A.C. et al. Effects of outdoor rearing and sire breed (duroc or yorkshire) on carcass composition and sensory and technological meat quality. Meat Science, v.45, n.1, p.1-15, 1997. Disponível em: <http://dx.doi.org/10.1016/S03091740(96)00101-5>. Acesso em: 26 jun. 2011. doi: 10.1016/ S0309-1740(96)00101-5.

GADE, P.B. Effect of rearing system and mixing at loading on transport and lairage behaviour and meat quality: comparison of outdoor and conventionally raised pigs. Animal, v.2, n.6, p.902-911, 2008. Disponível em: <http://dx.doi.org/10.1017/ S1751731108002565>. Acesso em: 21 jun. 2011. doi: 10.1017/ S1751731108002565.

GENTRY, J.G. et al. Alternative housing systems for pigs: Influences on growth, composition, and pork quality. Journal of Animal Science, v.80, p.1781-1790, 2002a. Disponível em: <http://jas.fass.org/content/80/7/1781.full>. Acesso em: 25 jun. 2011.
GENTRY, J.G. et al. Diverse birth and rearing environment effects on pig growth and meat quality. Journal of Animal Science, v.80, p.1707-1715, 2002b. Disponível em: <http:// jas.fass.org/content/80/7/1707.full>. Acesso em: 21 jun. 2011.

GENTRY, J.G. et al. Environmental effects on pig performance, meat quality, and muscle characteristics. Journal of Animal Science, v.82 p.209-217, 2004. Disponível em: <http:// jas.fass.org/content/82/1/209.full>. Acesso em: 26 jun. 2011.

GUY, J. H. et al. Growth performance and carcass characteristics of two genotypes of growing-finishing pig in three different housing systems. Animal Science, v.74, p.493-502, 2002. Dispoível em: <http//www.bsas.org.uk/Publications/Animal.../ pdf $>$. Acesso em: 25 jun. 2011. doi: 1357-7298/02/ $11180493 \$ 20 \cdot 00$.

HANSEN, L.L. et al. Effect of organic pig production systems on performance and meat quality. Meat Science, v.74, p.605615, 2006. Disponível em: <http://dx.doi.org/10.1016/ j.meatsci.2006.02.014>. Acesso em: 25 jun. 2011. doi: 10.1016/j.meatsci.2006.02.014.

HOFFMAN, L.C. et al. The growth and carcass and meat characteristics of pigs raised in a free-range or conventional housing system. South African Journal of Animal Science, v.33, n.3, p.166-175, 2003 . Disponível em:<http:// www.sasas.co.za/Sajas.html>. Acesso em: 26 de jun. 2011.

HOGBERG, A. et al. Fatty acid composition and tocopherol concentrations in muscle of entire male, castrated male and female pigs, reared in an indoor or outdoor housing system. Meat Science, v.68, p.659-665, 2004. Disponível em: <http:/ /dx.doi.org/10.1016/j.meatsci.2004.06.001>. Acesso em: 25 jun. 2011. doi: 10.1016/j.meatsci.2004.06.001.

JONSÄLL, A. et al. Sensory quality and cooking loss of ham muscle (m. Biceps femoris) from pigs reared indoors and outdoors. Meat Science, v.57, p.245-250, 2001. Disponível em: <http:/ /dx.doi.org/10.1016/S0309-1740(00)00097-8>. Acesso em: 21 jun. 2011. doi: 10.1016/S0309-1740(00)00097-8.

KELLY, H.R. et al. Effect of breed type, housing and feeding system on performance of growing pigs managed under organic conditions. Journal of the Science of Food and Agriculture, v.87, p.2794-2800, 2007. Disponível em: <http://dx.doi.org/ 10.1002/jsfa.3060>. Acesso em: 26 jun. 2011. doi: 10.1002/ jsfa.3060.

LAMBOOIJ, E. et al. Effects of housing conditions of slaughter pigs on some post mortem muscle metabolites and pork quality characteristics. Meat Science, v.66, p.855-862, 2004. Disponível em: <http://dx.doi.org/10.1016/j.meatsci.2003.08.007>. Acesso em: 21 jun. 2011. doi: 10.1016/j.meatsci.2003.08.007.

LEBRET, B. et al. Influence of outdoor rearing and indoor temperature on growth performance, carcass, adipose tissue and muscle traits in pigs, and on the technological and eating quality of dry-cured hams. Meat Science, v.62, p.447-455, 2002. Disponível em: <http://dx.doi.org/10.1016/S03091740(02)00036-0>. Acesso em: 24 jun. 2011. doi: 10.1016/ S0309-1740(02)00036-0.

LEBRET, B. et al. Influence of rearing conditions on performance, behavioral, and physiological responses of pigs to preslaughter handling, carcass traits, and meat quality. 
Journal of Animal Science, v.84, p.2436-2447, 2006. Disponível em: <http://jas.fass.org/content/84/9/2436.full>. Acesso em: 25 jun. 2011. doi: 10.2527/jas.2005-689.

LEBRET, B. Effects of feeding and rearing systems on growth, carcass composition and meat quality in pigs. Animal, v.2, n.10, p.1548-1558, 2008. Disponível em: <http://dx.doi.org/ 10.1017/S1751731108002796>. Acesso em: 26 jun. 2011. doi: $10.1017 / \mathrm{S} 1751731108002796$.

LOVATTO, P.A. et al. Meta-análise em pesquisas científicasenfoque em metodologias. Revista Brasileira de Zootecnia, v.36, p.285-294, 2007. Disponível em: <http://dx.doi.org/ 10.1590/S1516-35982007001000026>. Acesso em: 26 jun. 2011. doi: 10.1590/S1516-35982007001000026.

MILLET, S. et al. Performance, meat and carcass traits of fattening pigs with organic versus conventional housing and nutrition. Livestock Production Science, v.87, p.109-119, 2004. Disponível em: <http://dx.doi.org/10.1016/ j.livprodsci.2003.10.001>. Acesso em: 21 jun. 2011. doi: 10.1016/j.livprodsci.2003.10.001.

MILLET, S. et al. Performance and meat quality of organically versus conventionally fed and housed pigs from weaning till slaughtering. Meat Science, v.69, p.335-341, 2005. Disponível em: <http://dx.doi.org/10.1016/j.meatsci.2004.08.003>. Acesso em: 26 jun. 2011. doi: 10.1016/j.meatsci.2004.08.003.

NILZÉN, V. et al. Free range rearing of pigs with acess to pasture grazing- efect on fatty acid composition and lipid oxidation products. Meat Science, v.58, p.267-275, 2001. Disponível em: <http:// dx.doi.org/10.1016/S0309-1740(00)00164-9>. Acesso em: 21 jun. 2011. doi: 10.1016/S0309-1740(00)00164-9.

OLSSON, V. et al. Differences in meat quality between organically and conventionally produced pigs. Meat Science, v.64, p.287-297, 2003. Disponível em: <http://dx.doi.org/ 10.1016/S0309-1740(02)00200-0>. Acesso em: 21 jun. 2011. doi: 10.1016/S0309-1740(02)00200-0.
PUGLIESE, C. et al. Comparison of the performances of nero siciliano pigs reared indoors and outdoors. 1. Growth and carcass composition. Meat Science, v.65, p.825-831, 2003. Disponível em: <http://dx.doi.org/10.1016/j.meatsci.2004.02.020>. Acesso em: 26 jun. 2011. doi: 10.1016/j.meatsci.2004.02.020

PUGLIESE, C. et al. Performance of cinta senese pigs reared outdoors and indoors. 1. Meat and subcutaneous fat characteristics. Meat Science, v.69, n.3, p.459-464, 2004a. Disponível em: $<$ http://dx.doi.org/10.1016/j.meatsci.2004.09.001>. Acesso em: 21 jun. 2011. doi: 10.1016/j.meatsci.2004.09.001.

PUGLIESE, C. et al. Comparison of the performances of nero siciliano pigs reared indoors and outdoors: 2. Joints composition, meat and fat traits. Meat Science, v.68, p.523-528, 2004b. Disponível em: <http://dx.doi.org/10.1016/j.meatsci.2004.02.020>. Acesso em: 25 jun 2011. doi: 10.1016/j.meatsci.2004.02.020.

REY, A. I. et al. Feeding iberian pigs with acorns and grass in either free-range or confinement affects the carcass characteristics and fatty acids and tocopherols accumulation in longissimus dorsi muscle and backfat. Meat Science, v.73, p.66-74, 2006. Disponível em: <http://dx.doi.org/10.1016/ j.meatsci.2005.10.018>. Acesso em: 25 jun. 2011. doi: 10.1016/j.meatsci.2005.10.018.

STRUDSHOLM, K.; HERMANSEN, J.E. Performance and carcass quality of fully or partly outdoor reared pigs in organic production. Livestock Production Science, v.96, p.261268, 2005. Disponível em: <http://dx.doi.org/10.1016/ j.livprodsci.2005.02.008>. Acesso em: 21 jun. 2011. doi: 10.1016/j.livprodsci.2005.02.008.

TROMBETTA, M.F. et al. Influence of diet and rearing system on heavy pig performance, carcass and meat quality. Italian Journal of Animal Science, v.8, p.23-35, 2009. Disponível em: <http://dx.doi.org/10.4081/ijas.2009.23>. Acesso em: 24 jun. 2011. doi: 10.4081/ijas.2009.23. 